\title{
Multiple Linear Filling Defects in Coronary Artery: Differential Diagnosis
}

\author{
Hetan C. Shah ${ }^{10}$ M. Jyotsna² \\ 1 Padam Heart Care Center, Dadar, Mumbai, Maharashtra, India \\ ${ }^{2}$ NIMS, Punjagutta, Hyderabad, Telangana, India \\ Ind J Car Dis Wom 2021;6:277-280.
}

\begin{abstract}
Address for correspondence M. Jyotsna, MD, DM, FACC, FESC FICC, Chief Editor of IJCDW, President of WINCARS, HOD \& Professor of Cardiology, NIMS, Punjagutta, Hyderabad, Telangana, India (e-mail: janaswamyjyotsna@gmail.com).
\end{abstract}

\begin{abstract}
Keywords

- coronary artery

- linear filling

A 60-year-old male, diabetic, nonhypertensive, tobacco chewer, presented with a history of inferior wall myocardial infarction in 2007. Patient underwent percutaneous transluminal coronary angioplasty with stent to right coronary artery in 2007 , but no reports available. Now, patient was admitted with new onset crescendo angina for the last 1 month.
\end{abstract}

\section{Introduction}

A 60-year-old male, diabetic, nonhypertensive, tobacco chewer, presented with a history of inferior wall myocardial infarction in 2007. Patient underwent percutaneous transluminal coronary angioplasty with stent to right coronary artery (RCA) in 2007, but no reports available. Now, patient was admitted with new onset crescendo angina for the last 1 month (-Fig. $\mathbf{1}$ ).

Echocardiography shows no regional wall motion abnormality with preserved left ventricle (LV) systolic function.

Initial coronary angiogram ( - Fig. 2A and B) shows multiple filling defects in midsegment of dominant RCA followed by tight lesion.

\section{What Is the Diagnosis?}

\section{Opinion 1}

Presence of haziness reflected as multiple linear irregular contrast filling defects in RCA may be recanalized coronary thrombus. During acute thrombus state looks partial filling defects if it is a nonocclusive thrombus or filling defects proximally with no flow distally it is an occlusive thrombus. As the natural hemolytic system gets activated in the body after clot formation or because of thrombolytics, partial resolution of the thrombus occurs and when it persists it looks like this angiographic picture.

\section{Opinion 2}

Multiple linear irregular contrast filling defects may be complex atheromatous plaque-rupture/erosion including calcification. Of course, angiogram may reveal advanced lesions, plaque disruption, luminal thrombosis, and calcification, but other qualitative features of the underlying plaque cannot be assessed by this imaging technique.

\section{Opinion 3}

Multiple irregular contrast filling defects may be due to spontaneous coronary artery dissection (SCAD). This is an important differential diagnosis for partial recanalized thrombus, which requires optical coherence tomography (OCT) imaging of this RCA for confirmation. The diagnosis of SCAD is important as the management strategies also varies.

\section{Opinion 4}

Multiple irregular contrast filling defects may be due to insegment restenosis (ISR) in a previously stented patient. Usually, diffuse ISR may look eccentric or concentric and irregular narrowing than as filling defects. Intravascular ultrasound (IVUS) may be helpful in this case.
DOI https://doi.org/ $10.1055 / \mathrm{s}-0042-1742760$. ISSN 2455-7854. (c) 2022. Women in Cardiology and Related Sciences. All rights reserved.

This is an open access article published by Thieme under the terms of the Creative Commons Attribution-NonDerivative-NonCommercial-License, permitting copying and reproduction so long as the original work is given appropriate credit. Contents may not be used for commercial purposes, or adapted, remixed, transformed or built upon. (https://creativecommons.org/ licenses/by-nc-nd/4.0/)

Thieme Medical and Scientific Publishers Pvt. Ltd., A-12, 2nd Floor, Sector 2, Noida-201301 UP, India 


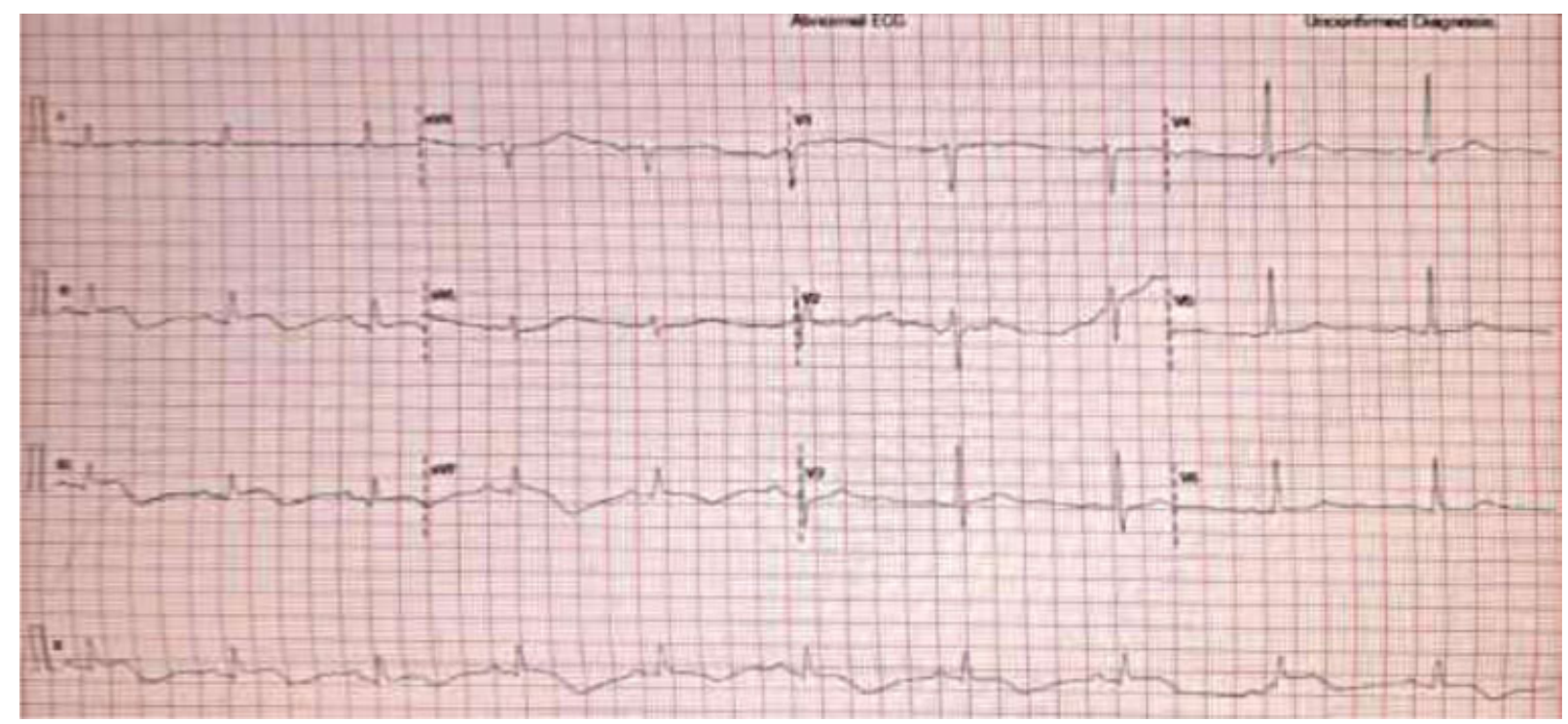

Fig. 1 Electrocardiogram at presentation revealed Inferior wall myocardial infarction.

\section{Opinion 5}

This type of coronary angiographic picture remotely may be coronary arteritis. Even though there are no specific diagnostic features of arteritis, few may be suggestive like tapered smooth narrowing has been described in cases of giant cell arteritis, skip lesions in Takayasu's arteritis and beads on a string or nodular appearance due to multifocal aneurysms in polyarteritis nodosa.

\section{Expert Opinion}

Major limitation of coronary angiography is in case of multiple linear filling defects or haziness diagnosis is not



Fig. 2 (A) Multiple filling defects in midsegment of dominant right coronary artery followed by tight lesion-left anterior oblique view. (B) Multiple filling defects in midsegment of dominant right coronary artery followed by tight lesion-lateral view. specific. Coronary imaging either with $\mathrm{OCT}^{1}$ or IVUS may help in characterizing the coronary lesion of this type.

What type of imaging is preferred in this case?

\section{Opinion 1}

Intracoronary imaging with IVUS and/or OCT is a useful tool to diagnose and characterize multiple luminal filling defect lesions and their functional significance. OCT is more powerful than IVUS in diagnosis of complex coronary lesions due to its high resolution and deeper penetration power compared to IVUS.

\section{Opinion 2}

OCT plays a very important role in identifying the lesion characteristic of multiple filling defects whose differential diagnoses include coronary arteritis, spontaneous coronary dissection with intraluminal thrombus. Studies have demonstrated that old coronary thrombi undergo gradual recanalization associated with neovascularization. This correlation has been strengthened by OCT studies that demonstrate excellent correlation of histopathology samples with coronary imaging techniques.

\section{Further Case Details}

First balloon dilatation is required to pass the OCT. The OCT imaging of RCA was performed on an $6 \mathrm{~F}$ guide cathetercompatible DRAGONFLY OPTIS (Abbott vascular, United States). - Fig. 3 showed the features of partially recanalized honeycombed thrombus ${ }^{2}$ and -Fig. 4 showed similar features in zoomed view. - Fig. 4 showed dissection in the distal lesion after balloon dilatation.

Percutaneous coronary intervention (PCI) of RCA was done successfully with stents covering both partially recanalized thrombus and distal lesion with dissection (-Fig. 5). ${ }^{3}$ 


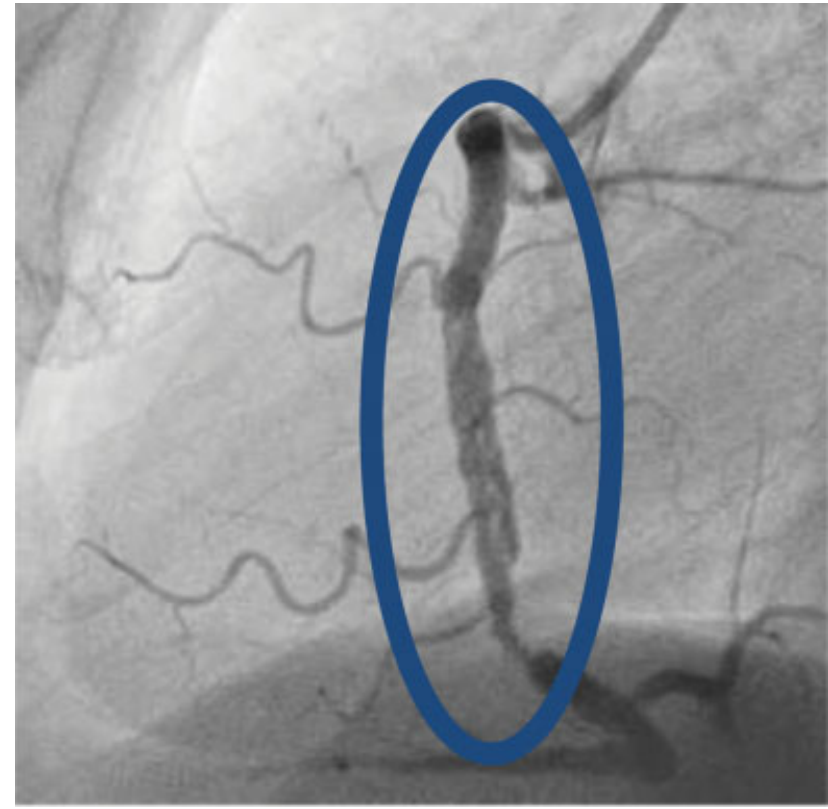

Fig. 3 Optical coherence tomography (OCT) finding showed a partially recanalized honeycombed thrombus in mid-right coronary artery (RCA) with a flap dissection over the distal RCA at the balloon dilatation site with $80 \%$ long tubular lesion in mid- and distal-RCA.

Post-procedure OCT showed good approximation of stents (-Fig. 6).

Patient was discharged on dual antiplatelet therapy and on 3 months follow-up no evidence of inducible ischemia was found.

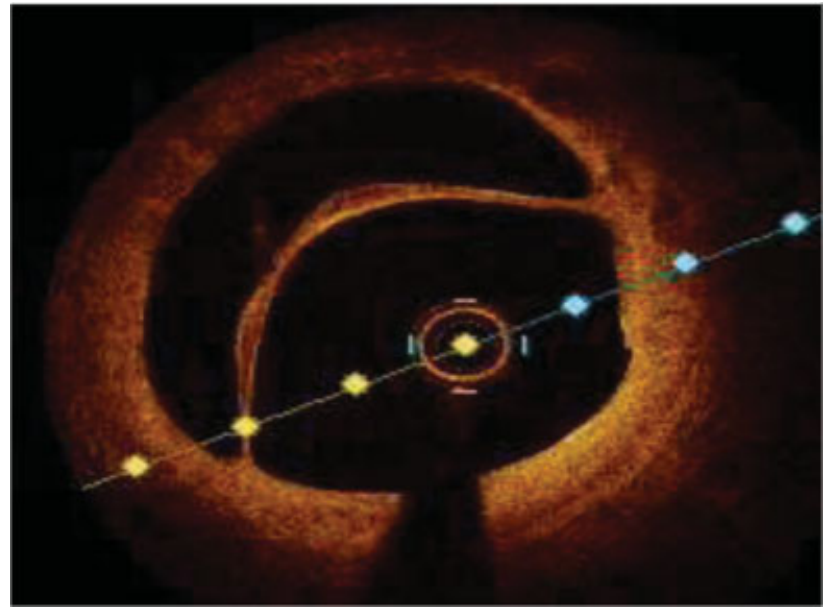

Fig. 5 Based on the findings and large area of ischemia and dominant right coronary artery coronary circulation, percutaneous coronary intervention with balloon angioplasty and two drug-eluting stents (DES) (XIENCE PRIME $4 \times 38 \mathrm{~mm}$ DES distally and XIENCE PRIME $4 \times 23 \mathrm{~mm}$ DES proximally) was done successfully.

\section{Final Diagnosis}

Ischemic heart disease, old inferior wall myocardial infarction, post-PCI stent to PDA (2007), recent onset angina, single vessel disease - multiple linear filling defects RCA (OCT diagnosis of spontaneous recanalization of coronary thrombus (SRCT) with good LV function and coronary risk factors diabetes mellitus and tobacco chewer.

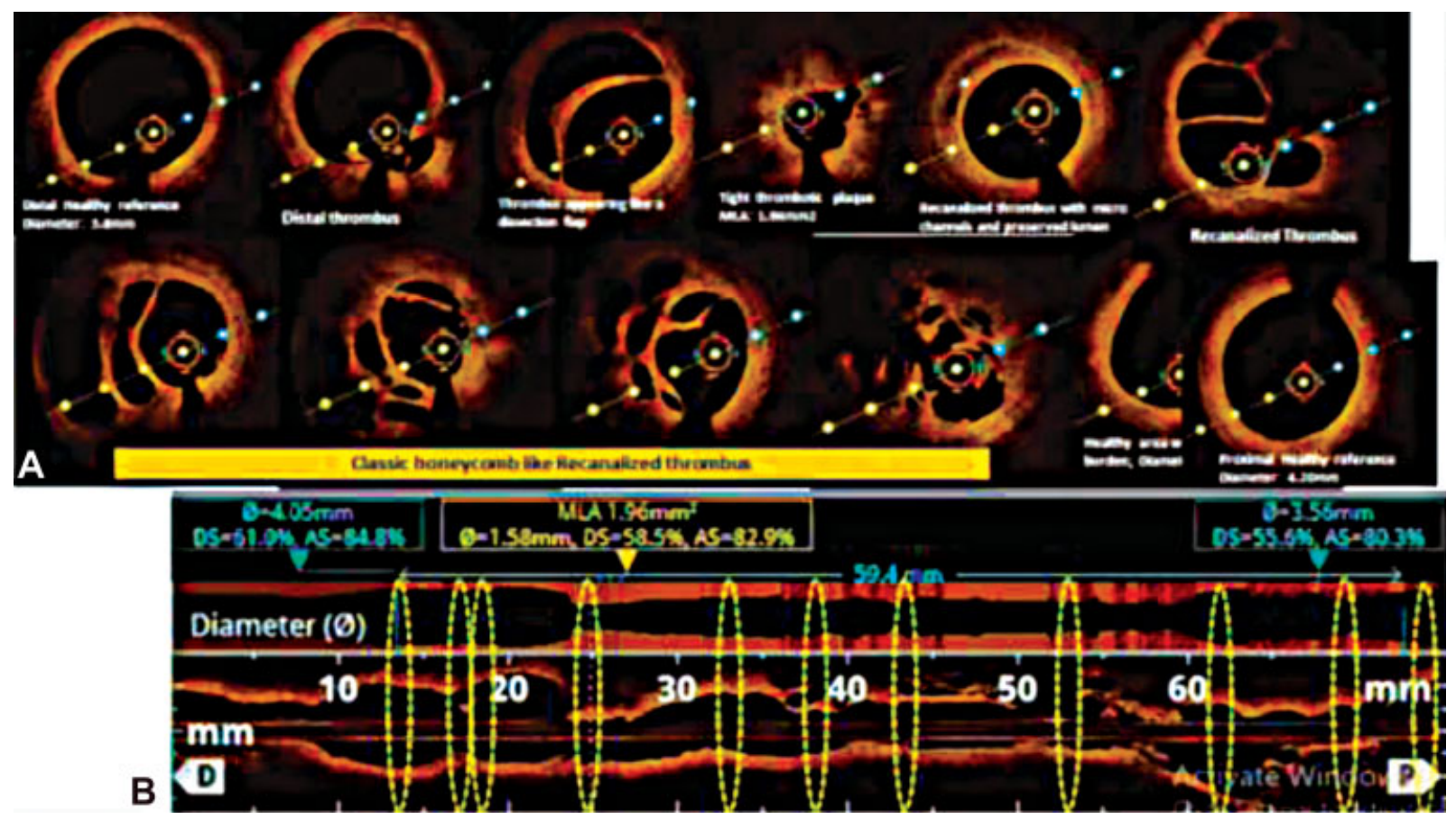

Fig. 4 Flap dissection (A) with multiple intraluminal channels of various sizes with honeycomb like appearance or lotus root like appearance or Swiss cheese like pattern noted (B). 


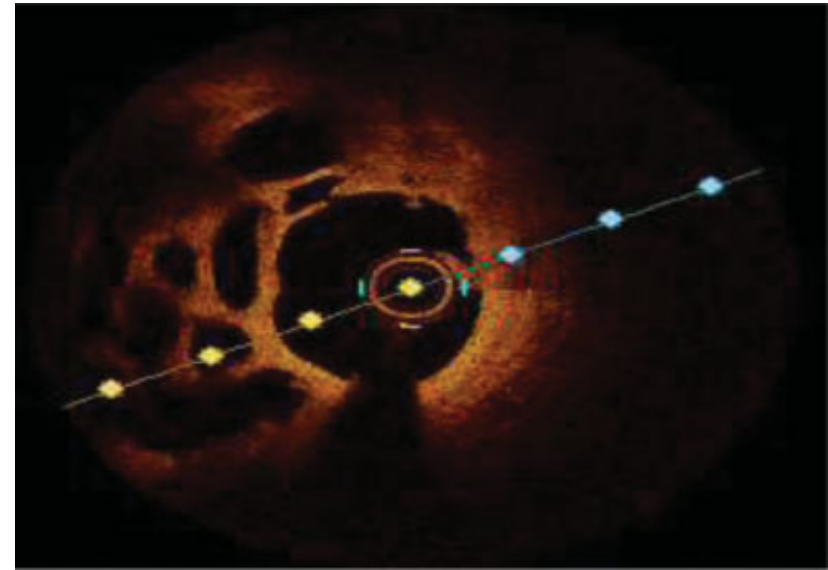

Fig. 6 Post-percutaneous coronary intervention optical coherence tomography showed enlargement of luminal area with well apposed stent and no dissection/thrombus.

\section{Discussion}

SRCT is a rare finding in patient with coronary artery disease usually as a result of evolution of coronary occlusion. Most of the time SRCT is underestimated on conventional angiography. On angiography, findings are suggestive of multiple linear filling defects or haziness or braided/pseudodissected filling defect. "Lesions of SRCT look borderline on angiogram but OCT identify its functional significance". As OCT has high spatial resolution $(10-15 \mu \mathrm{m}),{ }^{4}$ honeycomb like structure, lotus root appearance, or Swiss cheese pattern are unique features of SRCT termed on OCT images. Etiology of SRCT could be recanalization of a thrombus (in-situ/embolic) or Kawasaki disease. It must be differentiated from woven coronary artery (WCA)-a congenital anomaly in asymptomatic person with normal coronary blood flow 2 . On imaging, patient with WCA has intact vascular integrity, whereas SART has presence of thrombus, dissection, plaque erosion, or rupture. Management of SRCT depends on evidence of significantly large area of ischemia that is treated by coronary intervention in the form of angioplasty and stent. OCT plays very important role in decision-making process. ${ }^{5}$
By accurate lesion assessment and to avoid post-PCI suboptimal result like mal- position/edge dissection or stent under expansion. Hence, OCT enhances post-PCI immediate and late outcome. Only disadvantage of OCT is it increases procedure time, contrast volume, and pre-procedural complication. But on risk-benefit ratio scale, OCT helps not only in diagnosis of SRCT and its functional significance but also helps in identifying pathology of coronary wall whether it is dissection of atherosclerotic plaque or spontaneous coronary dissection of normal coronary wall. With the help of imaging, we can have better understanding of underestimated coronary lesions and planning for their customized treatment strategy. ${ }^{3,6}$

Conflict of Interest

None.

\section{References}

1 Vincent F, Ledermann B, Ricci JE, Cayla G, Lattuca B. Recanalized coronary thrombus: role of OCT in identifying a slow-evolving and underestimated coronary lesion. JACC Case Rep 2020;2(15): 2411-2413

2 Lin M, Su Z, Li J, Nie R, Wang J. Honeycomb-like structure in the right coronary artery treated with a drug-eluting stent: a case report and literature review. J Int Med Res 2018;46(05): 2008-2013

3 Spinu M, Homorodean C, Ober MC, Olinic M, Mircea PA, Olinic DM. Optical coherence tomography for in vivo identification, characterization and optimal treatment of spontaneous recanalization of coronary thrombus. In Vivo 2020;34(02):745-756

4 Kang S-J, Nakano M, Virmani R, et al. OCT findings in patients with recanalization of organized thrombi in coronary arteries. JACC Cardiovasc Imaging 2012;5(07):725-732

5 Cho JM, Raffel OC, Stone JR, Kim CJ, Jang IK. Spontaneous recanalization of a coronary artery after thrombotic occlusion: in vivo demonstration with optical coherence tomography. J Am Coll Cardiol 2010;55(12):1274. Doi: 10.1016/j.jacc.2008.11.071

6 Vijayvergiya R, Krishnappa D, Kasinadhuni G, Gupta A, Panda P, Ratheesh KJ. Coronary dissection or a recanalized thrombus? Optical coherence tomography has the answer. IHJ Cardiovascular Case Reports (CVCR) 2018;2(01):6-8 